\title{
Collective Intelligence in Computer-Mediated Collaboration Emerges in Different Contexts and Cultures
}

\author{
David Engel \\ Massachusetts Institute of \\ Technology \\ Cambridge, USA \\ dengel@mit.edu
}

\author{
Christopher F. Chabris \\ Union College \\ Schenectady, USA \\ chabris@gmail.com
}

\author{
Carolin Kaiser \\ University Erlangen-Nuremberg \\ Nuremberg, Germany \\ carolin.kaiser@gfk-verein.org
}

\author{
Anita Williams Woolley \\ Carnegie Mellon University \\ Pittsburgh, USA \\ awoolley@cmu.edu
}

\author{
Masamichi Takahashi \\ Fuji Xerox Co., Ltd. \\ Kanagawa, Japan \\ masamichi.takahashi@fujixerox.co.jp
}

Young Ji Kim
Massachusetts Institute of
Technology
Cambridge, USA
youngji@mit.edu

\author{
Ishani Aggarwal \\ Tilburg University \\ Tilburg, Netherlands \\ ishaniaggarwal@gmail.com
}

\author{
Keiichi Nemoto \\ Fuji Xerox Co., Ltd. \\ Kanagawa, Japan \\ keiichi.nemoto@fujixerox.co.jp
Thomas W. Malone
Massachusetts Institute of
Technology
Cambridge, USA
malone@mit.edu

\begin{abstract}
Collective intelligence (CI) is a property of groups that emerges from the coordination and collaboration of members and predicts group performance on a wide range of tasks. Previous studies of CI have been conducted with lab-based groups in the United States. We introduce a new standardized online battery to measure CI and demonstrate consistent emergence of a CI factor across three different studies despite broad differences in (a) communication media (face-to-face vs online), (b) group contexts (shortterm ad hoc groups vs long-term groups) and (c) cultural settings (US, Germany, and Japan). In two of the studies, we also show that CI is correlated with a group's performance on more complex tasks. Consequently, the CI metric provides a generalizable performance measure for groups that is robust to broad changes in media, context, and culture, making it useful for testing the effects of general-purpose collaboration technologies intended to improve group performance.
\end{abstract}

\section{Author Keywords}

Collective intelligence; group performance; online collaboration; cross culture; factor analysis; outcome metrics.

\section{INTRODUCTION}

Recent research on group performance demonstrates that

Permission to make digital or hard copies of all or part of this work for personal or classroom use is granted without fee provided that copies are not made or distributed for profit or commercial advantage and that copies bear this notice and the full citation on the first page. Copyrights for components of this work owned by others than ACM must be honored. Abstracting with credit is permitted. To copy otherwise, or republish, to post on servers or to redistribute to lists, requires prior specific permission and/or a fee. Request permissions from Permissions@acm.org.

CHI 2015, April 18 - 23, 2015, Seoul, Republic of Korea Copyright 2015 ACM 978-1-4503-3145-6/15/04 ..\$15.00 http://dx.doi.org/10.1145/2702123.2702259 there is a single statistical factor for groups, just as there is for individuals, that predicts how well a group will perform on a wide range of different tasks [43]. This factor, called collective intelligence (CI), depends not only on the characteristics of the individuals in the group but also on how they work together. In a sense, therefore, this factor focuses on processes that are critical for using computers to help groups of humans interact.

An important type of research in human-computer interaction and computer-supported cooperative work involves empirically studying how well various kinds of computer tools help groups of people work together cooperatively. Typically, this is done by choosing a specific task and evaluating how well groups perform the task with (and sometimes without) the computer tools. However, the generalizability of such studies is always limited by the nature of the specific task chosen.

But imagine that we had some general way of measuring the ability of a group to work together, regardless of the type of task involved. Or, more precisely, what if we could measure the performance of a group working together on a carefully chosen set of tasks and then use these measurements to statistically predict how well the group would perform on a very wide range of other tasks?

Here we introduce a generalizable measure of team collective intelligence which can do just this. For example, web conferencing systems can be used to support groups performing many kinds of tasks, but it isn't obvious how to pick the tasks to use for testing such systems. Our collective intelligence test battery provides a carefully 
chosen set of tasks, which can be used to test the effects of such systems on group performance, and a statistical basis for using these results to predict performance on a much wider range of other tasks.

In this paper, we explore the generality of our newly designed computer-based measure of collective intelligence $[8,43]$. We meta-analyze three data sets to test the degree to which the CI factor emerges not only across different tasks, but across communication media (face-to-face vs. online), group contexts (short-term ad hoc groups vs long-term groups) and cultures (US, Germany, Japan) making this test battery generalizable to a variety of settings.

\section{BACKGROUND}

\section{Measuring Collective Intelligence}

We are increasingly reliant on groups in a growing number of domains in our daily lives. Decisions in healthcare, science, law enforcement, national security, transportation, and public policy, to name just a few, are all increasingly made by groups of highly networked individuals, and their performance is driven not only by their individual skills, but also by their ability to collectively collaborate and coordinate. However, until recently, we have lacked good tools to evaluate the degree to which groups can consistently perform at a high level.

By contrast, in research on individual performance, over the last century psychological researchers have devised effective tools for predicting who is likely to perform at a high level across many domains and over time. Cognitive psychologists have repeatedly demonstrated that a single statistical factor-often called "general intelligence" or "g" - emerges from the correlations among individuals' performance on a wide variety of cognitive tasks $[6,38]$ and is highly predictive of individuals' future performance. Recently, Woolley et al. [43] applied the same statistical techniques used in individual intelligence research to measuring the intelligence of groups. The researchers first gave 192 groups a wide range of tasks that required qualitatively different collaboration processes for successful accomplishment [24]. In a factor analysis of all the groups' scores, the first factor accounted for $43 \%$ of the variance in performance. This is consistent with the $30-50 \%$ of variance typically explained by the first factor in a battery of individual cognitive tasks [4]. In individuals, this factor is called "intelligence" or " $g$ ". For groups, Woolley et al. referred to it as "collective intelligence" or " $c$," and it is a measure of the general effectiveness of a group on a wide range of tasks. The $c$ factor also predicted how the groups performed on more complex tasks at a later point in time, above and beyond the predictive ability of the average individual intelligence of group members. Woolley et al. also showed that a group's collective intelligence was positively affected by the proportion of women in the group, the group members' social sensitivity, and their equal participation in group discussion.
The emergence of a single collective intelligence factor is itself a somewhat surprising outcome. The null hypothesis of any factor analysis is the emergence of as many factors as there are scores entered into the analysis. In other words, a test battery including five tasks should yield five roughly equivalent factors, unless there is a common force driving performance across some or all of them. And indeed, in the history of research on teams, much attention has been given to the different demands of different sorts of tasks, and the development of taxonomies to articulate the different ways groups would need to collaborate to successfully accomplish them. This would suggest that teams that perform well on one kind of task would not necessarily perform well on others. However, the emergence of a single dominant collective intelligence factor strongly suggests a central mechanism or set of mechanisms driving team performance across many domains. This has important implications for designing technological systems to support group work, as those that will enhance these key underlying collective abilities will have a much more profound effect on collaboration more generally.

This approach to conceptualizing and measuring intelligence in groups builds and improves upon traditional approaches to investigating intelligence and performance in team settings in two important ways. First, the relationship between team cognitive ability and performance has been previously examined as the aggregate of individual member intelligence. Individual intelligence has been shown to be a moderate predictor of team performance in lab-based settings, but the correlation of team average cognitive ability and performance becomes much smaller in field settings $[7,18,26]$. One probable explanation for this lack of explanatory power is the attempt to conceptualize team ability by aggregating individual-level measurements, which loses information in generalizing across levels of analysis [32].

Second, the vast majority of team research measures performance on a single task, whereas most teams in real world settings need to perform a multitude of tasks to operate effectively. Thus, a more robust method for capturing what constitutes team collective intelligence, utilizing uniquely team-level information and observation of performance on a variety of tasks will improve our ability to predict which teams are likely to consistently perform at a high level in the future.

Though the existence and emergence of a general collective intelligence factor in teams has been empirically supported [43], the existing work relies on U.S. lab-based samples involving groups of strangers collaborating face-to-face for a few hours. Indeed, recent research examining CI in a small sample of distributed groups raised questions about whether a single CI factor exists in such settings [1]. This is an important issue to explore, as work in organizations, both traditional and online, usually involves individuals with an ongoing relationship with each other and/or a 
common institution, collaborating both face-to-face and via media, from around the globe. Will a collective intelligence factor emerge in settings with these characteristics? Or will features of the culture, context, or the relationships among members create so much variance or randomness in how groups perform different tasks that a collective intelligence factor will not emerge?

\section{Group Collaboration Online vs. Face-to-Face}

Well over half of all employees in organizational settings have at some point worked in online teams [22]. Online teams are used in almost all industries and in a variety of areas, such as software development, customer service, sales and $R \& D[3,11,23]$.

A commonly addressed issue in online group collaboration is the lack of nonverbal and social cues during interaction. Early media characteristics theories suggested that the lack of cues in computer-mediated communication (CMC) makes it inferior to face-to-face communication in facilitating social interactions and conveying more complex, equivocal messages [5][36]. According to this view, technologies that do not offer the transmission of nonverbal cues cannot adequately support the processing of socioemotional and richer content, thereby inhibiting group members from engaging in further interaction. When group members collaborate on a task, limited interaction can potentially prevent them from exchanging necessary information to perform tasks effectively.

However, this so-called "cue-filtered-out" perspective, which claims face-to-face collaboration is better than textbased $\mathrm{CMC}$, has been broadly challenged. In particular, Walther's social information processing theory provided a major counterargument to the early theories, by positing that with time, individuals are able to adapt to the lack of nonverbal cues, and engage in social and relational communication online just as well as face-to-face [42]. In addition, in some cases, text-based computer-mediated communication has been argued to be "superior" to face-toface in that it gives individuals more agency and control in self-presentation by selectively transmitting cues that portray them in a positive light [21].

In spite of this, when a group works together online only, the group's ability to perform tasks may reflect not only their collective intelligence but also the degree to which members utilize and adapt to online collaboration tools (e.g., chat, real-time collaborative editing software) in task performance. Groups may differ in their experience with a given medium, which may emerge as a potential factor that creates variation in groups' performance independent of their collective intelligence [1]. Given these arguments, it is not clear whether a general collective intelligence factor will emerge to describe a group's performance capability regardless of communication media that support the group collaboration.

\section{Group Collaboration in Short-Term Ad Hoc Groups vs Long-Term Groups}

Woolley et al. [43] found the initial evidence of collective intelligence in groups of strangers gathered for a lab study. Would this finding apply to groups with ongoing work relationships? Literature that provides a basis for the linkage between time and group collaboration suggests mixed scenarios. On one hand, it is plausible to think that long-term groups may have an advantage in developing rules of coordination compared to ad hoc short-term groups. Ad hoc groups, without a stabilized work routine, may require more time for coordination, which can hurt performance [39]. In addition, long-term groups may be able to develop more trust among members of the group, promoting smooth information sharing and knowledge transfer [19]. Thus, one may expect greater consistency in performance across different types of tasks and thus the emergence of collective intelligence for long-term groups.

On the other hand, there can be a downside to longer tenure in groups. Groups can fall into habitual routines that they apply in inappropriate situations [9]. In addition, groups with ongoing relationships are subject to relational factors biasing information exchange such that members seek information only from someone they know and have access to [2]. This may lead to inconsistencies in how the same group performs different tasks, reducing the degree to which a CI factor would predict performance across domains. Therefore, one may imagine that collective intelligence emerges in a more consistent fashion in taskoriented short-term groups as they focus on a given task under time constraints, preventing existing relationships and prior assumptions about group members from affecting task-oriented interaction.

\section{Group Collaboration across Cultures}

Culture influences what social norms individuals recognize and what behaviors they believe they should or should not engage in when collaborating with others [30,41]. Culture is a multi-dimensional construct, and a number of competing frameworks have been proposed for thinking about how cultures differ from one another [12] (see [25] for a review). Some view cultural differences in terms of core values, which are stable across individuals, situations, and time [13][29], whereas others take a systems perspective, seeing culture as a dynamic system shaped by sociocultural artifacts such as symbols, discursive routines, and social institutions and structures [15]. We acknowledge that both psychological processes and sociocultural practices shape culture, resulting in both commonality and variability within a national culture. While not every member of one national culture thinks and behaves alike during group collaboration, culture has been considered an undeniable factor that explains differences in understanding of groups and collaboration [12][34].

To understand possible sources of cross-cultural differences in collective intelligence, if any, we discuss two dimensions that have been demonstrated to be particularly relevant to 
computer-mediated group communication, and potentially relevant to collective intelligence: individualism vs. collectivism [13], and high- versus low-context cultures [10].

Individualism-collectivism is an analytical dimension that captures the relative importance people accord to personal interests and to shared pursuits. Individualism is the condition in which personal interests are accorded greater importance than are the needs of groups [13,41]. Individualists look after themselves and tend to ignore group interests if they conflict with personal desires. Collectivism, on the other hand, occurs when the demands and interests of groups take precedence over the desires and needs of individuals. Collectivists look out for the wellbeing of the groups to which they belong, even if such actions sometimes require that personal interests be disregarded.

High-vs. low context culture is an additional distinction that often parallels individualism-collectivism [10]. A highcontext culture is one in which people are deeply involved with each other. As a result of intimate relationships among people, a structure of social hierarchy exists, individual inner feelings are kept under strong self-control, and information is widely shared through simple messages with deep meaning. A low-context culture is one in which people are highly individualized, somewhat alienated, and fragmented, and there is relatively little involvement with others. Society in general imposes less on individuals' lives, and communication between people is more explicit and impersonal.

It is conceivable that notions of collective intelligence developed based on Western samples (made up of largely individualistic and low-context cultures) would not generalize to high-context, collectivistic cultures such as those found in East Asia. We might observe less variance in general in how more collectivistic teams perform, given their commitment to the group, making the notion of a predictive measure of collective intelligence one that has less utility for teams in collectivistic societies. Or, if we employ a traditional approach of convening lab-based groups of strangers, we might find in high context cultures a difficulty for such groups to "gel" enough to exhibit a consistent level of performance across tasks. Thus the degree to which notions of collective intelligence will generalize at all to high context, collectivistic samples remains an open question.

The difficulties of extending the collective intelligence construct to high context, collectivistic cultures may be even greater when groups collaborate online. Other research has predicted and found that high-context, collectivistic cultures, such as those in East Asia, place more value than do low-context cultures on feedback and social cues that may be reduced or eliminated in some forms of computermediated communication. For example, Chinese collaborators have been shown to engage in dramatically less communication when conversations are computermediated than face-to-face, compared to American collaborators who exhibit little to no reduction in communication between the two modes [35]. By contrast, improvements to computer mediated communication, such as multi-party chat, emoticons, and audio-video chat, which enhance communicators ability to convey and respond to context, are much more broadly embraced by high-context, collectivistic cultures than low-context, individualistic cultures [14]. Taken together, this suggests we might see a much weaker or less consistent collective intelligence factor emerge in online teams from high-context, collectivistic cultures (e.g., Japan) compared to their low-context, individualistic counterparts (e.g., Germany, US).

\section{METHODS}

\section{Test Design}

Following the protocol outlined in Woolley et al. [43], we adapted the original collective intelligence test battery into an online tool that enabled us to administer the test in a standardized way to all groups, whether working face-toface or online. All members could see the input of others in the real-time. Even in settings where all group members were collocated and could communicate freely, they worked together through the browser-based platform. Figure 1 shows a screenshot of the interface.

Expanding on the work from Woolley et al.[43], we aimed to develop a battery of tasks that span a wide and comprehensive range of group processes. Consequently, we chose the initial set of tasks based on well-established taxonomies of group tasks as those developed by McGrath [24] and Larson [17] together with other basic types of task groups proposed by researchers in cognitive psychology and artificial intelligence [28,33,37]. The design of the specific tasks and task groups was strongly inspired by the

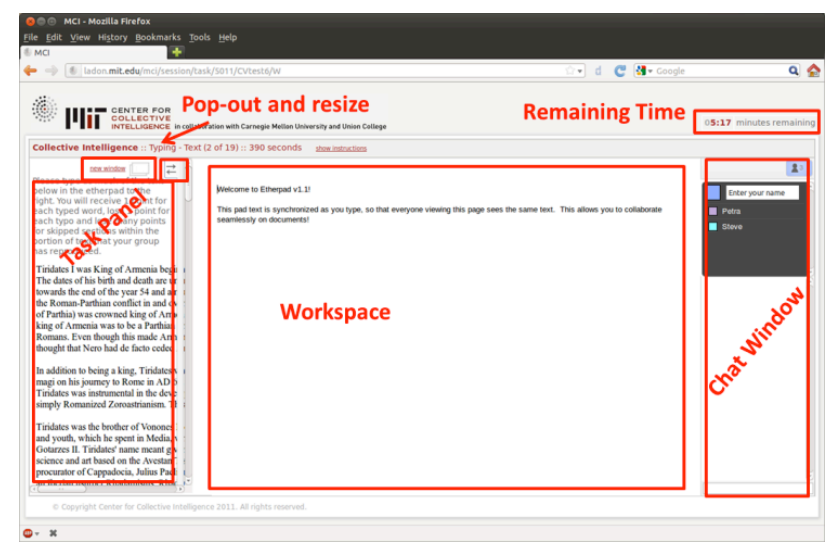

Figure 1: The online testing interface. The current task and instructions are displayed on the left hand side. The instructions can be popped out into a separate window. An integrated chat window on the right allows the groups to coordinate their efforts. The workspace in the center is a synchronized Etherpad that allows collaborative editing. 


\begin{tabular}{|c|c|c|}
\hline Task Group & Process & Summary \\
\hline Typing (TY) & Executing & $\begin{array}{l}\text { Create a copy of either a } \\
\text { text or a long list of } \\
\text { numbers as accurately as } \\
\text { possible. }\end{array}$ \\
\hline $\begin{array}{l}\text { Detection } \\
\text { (DE) }\end{array}$ & Sensing & $\begin{array}{l}\text { Study a large grid of images } \\
\text { or words and answer } \\
\text { questions about them such } \\
\text { as naming the most frequent } \\
\text { object in that grid }\end{array}$ \\
\hline Memory (ME) & Remembering & $\begin{array}{l}\text { Collectively remember } \\
\text { enough about videos, } \\
\text { images or words to answer } \\
\text { specific questions }\end{array}$ \\
\hline Judgment (JG) & Choosing & $\begin{array}{l}\text { Make a judgment such as } \\
\text { estimating how a larger } \\
\text { population of people would } \\
\text { rate a slogan or an image }\end{array}$ \\
\hline $\begin{array}{l}\text { Brainstorming } \\
\text { (BS) }\end{array}$ & Generating & $\begin{array}{l}\text { Come up with as many } \\
\text { words for a specific pattern, } \\
\text { uses for an object (e.g. a } \\
\text { brick) or equations } \\
\text { following some constraints }\end{array}$ \\
\hline $\begin{array}{l}\text { Matrix } \\
\text { Solving (MS) }\end{array}$ & Choosing & $\begin{array}{l}\text { Solve matrix puzzle } \\
\text { problems }\end{array}$ \\
\hline $\begin{array}{l}\text { Unscramble } \\
\text { Words (SC) }\end{array}$ & Choosing & $\begin{array}{l}\text { Figure out what word is } \\
\text { represented in a set of } \\
\text { letters which have been } \\
\text { randomly shuffled around }\end{array}$ \\
\hline
\end{tabular}

Table 1:Tasks used in our Collective Intelligence (CI) test battery, the group process tested and a brief explanation.

set of tasks used in the original study of collective intelligence [43]. The tasks in the online battery fall into five general categories of group ability:

a) Generating: Requires the generation of new information

b) Choosing: Groups have to jointly pick one answer among a set of possible choices. The question can either have one objectively correct answer or be based on subjective ratings.

c) Executing: Requires careful coordination of psychomotor skills of team members such as joint typing.

d) Remembering: Groups need to remember features of previously presented information requiring them to distribute their attention during the presentation phase and later integrate their knowledge, similar to ideas explored in transactive memory research [11].

e) Sensing: Requires groups to recognize patterns in a noisy stimulus that is calibrated to exceed the capabilities of an individual.

We aim to have little overlap between task groups with respect to the specific group ability or process they are measuring. We avoided more complex, composite tasks since for those, the intertask correlations could stem from similarities between the tasks, instead of an underlying factor driving performance across all of them. Table 1 provides a brief explanation of the tasks deployed in the different task groups.

Most of these task groups contain several similar tasks in order to create a reliable index of the category. For example, the Memory task group contains a video memory task in which the members as a group need to remember certain features of a short movie clip, an image memory task where the group has to memorize a picture or a set of pictures and a word memory task in which the objective is to reproduce a previously presented and memorized list of words. We also aim to include both verbal and nonverbal operationalizations of each task group.

We are reporting meta-analyses of data from three studies conducted over a timespan of two years. Between the times when the studies were conducted, we not only evolved the tool but also improved several of the tasks. The subsets of tasks that were used in each study differ slightly, due to this evolution, and to specific restrictions such as language, time constraints, and concerns about possible cheating.

Given differences in timing of administration and cultural setting, there were minor differences in the specific task combinations the groups had to solve and differences in the wording especially across different languages. However, this does not inhibit our ability to explore the main question of our study, which is to what degree does group context and culture affect the emergence of a collective intelligence factor? Japan and Germany are particularly interesting contrasts for our purposes as they are on opposite ends of the cultural spectrum in terms of individualism/collectivism and high/low context.

\section{Cl Battery Administration and Scoring}

The group members were assigned to groups either at random or according to a pre-existing team membership (as described for each specific study below.) We provided each person with an anonymized login ID that they used to login to our testing server and join their group. Once all group members were logged in, our system started the administration of the tasks. The system continuously provided a collaborative environment for online task solving where group members were presented with the task instructions, a joint workspace and a chat interface (see Figure 1). The system recorded answers and interactions, 
and moved the group members simultaneously through the tasks according to pre-specified time allocations.

After the teams had completed the online battery, we downloaded the results and scored the tasks with the help of a semi-automated script. As mentioned above, some of the task groups contained multiple subtasks (e.g. the memory task group containing a video, an image and a word memory task). We combined these tasks by averaging the normalized subscores.

Finally, we conducted a principal component analysis to explore the factor structure of the scores in each study. The score of a group on the first factor is what constitutes the group's collective intelligence (CI) score.

\section{Samples and Measures}

The three studies took place in different settings, different cultures, and different languages; included groups with varying lengths of working relationships; and contained different modes of communication between the group members. All groups completed our online, browser-based test described above which was the basis for their CI score.

\section{Study 1: United States}

The first study contained 68 groups of 4 people that were recruited from the general population in the Boston area using online advertisements. The team members were compensated for their participation. All groups were invited to a lab setting where they completed a one-hour battery of tests as a team. Participants were randomly assigned to groups. Approximately half of the groups worked online and communicated solely through the text-based chat window provided by our online tool. The other half were allowed to communicate normally while seated around a table to work on the battery of tasks. After completing the online collective intelligence test, all teams also participated together in the Desert Survival Situation task [16]. The Desert Survival Situation task is a well-established team exercise where a group is asked to imagine a hypothetical scenario of a plane crash in a desert and to rank 15 items salvaged from the crash in order of their importance to the group's survival. The task requires an intense level of group interaction and a synergistic integration of various pieces of information, making it a suitable criterion task for collective intelligence. Other results from this study were reported by Engel et al. [8], and further details about the study procedure are reported there.

\section{Study 2: Germany}

In the second study, we recruited 116 groups of 2-5 students from a class on innovation management that was attended by students from the fields of business studies, economics, computer science, and engineering at a German university. The groups were intact teams that worked together on an extra-credit project for which they had to invent and prototype a smartphone app in the area of social service, education or nutrition. Their participation in our study was voluntary but was incentivized by a raffle of monetary prizes among all groups that completed the test battery. The team members knew each other and had previously worked together in the same configuration for earlier group assignments in the class. The groups were furthermore allowed to self-select their mode of communication while completing the CI battery, ranging from (a) face-to-face communication, through (b) ad hoc voice and video communication of their own choosing, to (c) the text chat facility built into our testing platform. For purposes of analysis, we considered teams communicating primarily by text, voice or video chat to be "online" and those that were primarily co-located to be "face to face." To reduce potential confusions and noise due to different levels of comfort with English, we had native German speakers translate the user-interface and the tasks in the battery into German before administration.

\section{Study 3: Japan}

In the third study, we recruited 25 groups of 4 persons through a temporary staffing agency in Japan and invited them to a lab setting to participate. They were compensated for their participation in a shortened battery. The setting restricted them to only communicate through the chat interface in our system. Native speakers translated the battery and the interface into Japanese. Because of time constraints in administering the battery and concerns about the translation of some of the verbally intensive tasks, we selected and administered only a subset of five task groups in this context.

\section{RESULTS}

1. A general Collective Intelligence factor emerges consistently in groups performing collaborative tasks in different cultural settings

In examining the subtests of any test of general intelligence, researchers want to see a small to moderate average pairwise correlation, which indicates an underlying relationship among the tests but sufficient independence that each is capturing unique variance. The pattern of pairwise correlations among the groups of tasks in the CI battery is displayed in Table 2. We see there an average pairwise correlation of 0.32 among the subtests of the collective intelligence battery for the US groups, of 0.23 for the Japanese groups, and of 0.37 for the German groups.

Next, we analyzed the factor structure underlying the group scores. The scree plot in Figure 2 shows the amounts of variance explained by each of the factors. Although the settings, methods of communication, and cultural norms differ substantially among the three studies, there is a striking similarity among the factor structures. Similar to classical results for individual intelligence tests, the first factor explains about $40 \%$ or more of the variance in all settings. Confirmatory factor analyses support the conclusion that a one factor model is the best fit for the data. In the U.S. sample: $\chi^{2}=28.36, p=.37$, RMSEA $=.01$. $\mathrm{AIC}=56.36$; in the German sample: $\chi^{2}=15.09, p=.37$, 


\begin{tabular}{|c|c|c|c|c|c|c|c|}
\hline & & TY & $\mathrm{BS}$ & MS & $\mathrm{ME}$ & $\mathrm{DE}$ & $\mathrm{SC}$ \\
\hline \multirow{6}{*}{$\begin{array}{l}\text { United } \\
\text { States }\end{array}$} & $\mathrm{BS}$ & .46 & & & & & \\
\hline & MS & .53 & .53 & & & & \\
\hline & $\mathrm{ME}$ & .42 & .27 & .45 & & & \\
\hline & $\mathrm{DE}$ & .35 & .31 & .28 & .22 & & \\
\hline & $\mathrm{SC}$ & .25 & .51 & .40 & .23 & .24 & \\
\hline & $\mathrm{JG}$ & .19 & .29 & .20 & .29 & .05 & .30 \\
\hline \multirow[t]{5}{*}{ Japan } & $\mathrm{BS}$ & .00 & & & & & \\
\hline & MS & .35 & .43 & & & & \\
\hline & $\mathrm{ME}$ & .21 & .26 & .04 & & & \\
\hline & $\mathrm{DE}$ & .22 & .52 & .16 & .07 & & \\
\hline & $\mathrm{SC}$ & .01 & .40 & .15 & .25 & .19 & \\
\hline \multirow[t]{6}{*}{ Germany } & $\mathrm{BS}$ & .45 & & & & & \\
\hline & MS & .46 & .35 & & & & \\
\hline & $\mathrm{ME}$ & .45 & .48 & .19 & & & \\
\hline & $\mathrm{DE}$ & .22 & .28 & .05 & .25 & & \\
\hline & $\mathrm{SC}$ & .41 & .40 & .20 & .41 & .17 & \\
\hline & JG & .19 & .24 & .20 & .18 & .07 & .14 \\
\hline
\end{tabular}

Table 2:Pairwise correlations for each task group for each study. See Table 1 for task group abbreviations. Correlations $>.20$ are significant at $p<.05$ in U.S. and Germany samples; > .40 in Japan sample. Note: The JG subtest was not included in the Japan study.

RMSEA $=.03, \mathrm{AIC}=43.09$; and in the Japanese sample: $\chi^{2}=9.18, p=.42, \mathrm{RMSEA}=.03, \mathrm{AIC}=33.18$.

A second observation that is of interest when we compare these studies is the congruence of the loadings of the different task groups on the main collective intelligence factor. Coefficients of congruence are useful for comparing the similarity of factor patterns [40]. Lorenzo-Seva and ten Berge [20] suggested "a value in the range .85-.94 corresponds to a fair similarity, while a value higher than .95 implies that the two factors or components compared can be considered equal." In the current samples, the loadings produce congruence coefficients that range from $\mathrm{r}_{\text {congruence }}=.94$ (Japan:German sample) to $\mathrm{r}_{\text {congruence }}=.99$ (German: US sample).

2. The Collective Intelligence factor is consistent across communication media and group contexts

In the US setting, we randomly assigned groups to two conditions [8]. In the online condition, group members were not able to see each other, and they communicated solely via the provided chat interface. In the face-to-face condition, subjects were seated around a conference table and could communicate freely by speaking to each other. By holding all other factors constant, such as test administration, subject pool, and location, this study

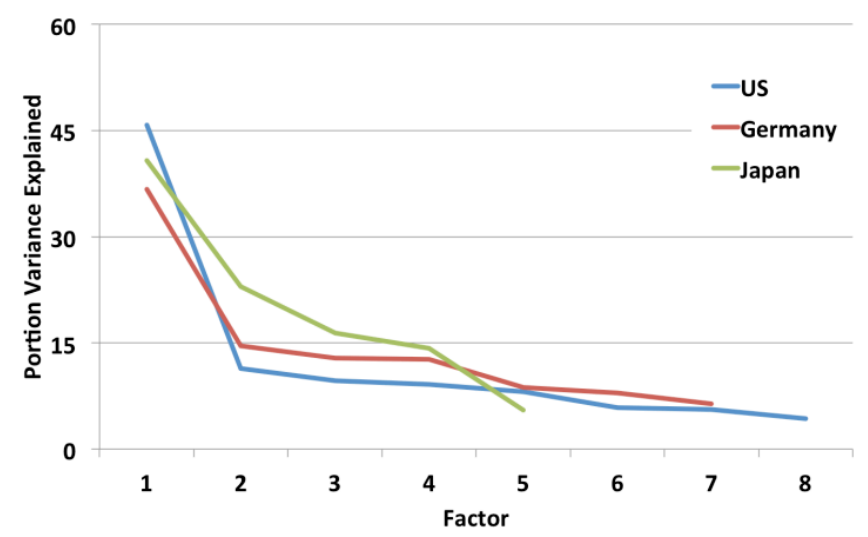

Figure 2: Scree plot of the variance explained by the factors in the three studies. The first factor explains around $40 \%$ of the variance while the next factors explain significantly less. The number of factors is equivalent to the number of task groups in the analysis. The German dataset had the highest variance in group sizes and modes of communication, which contributed to the slightly reduced variance explained by the first factor. The Japanese study contained only a subset of five task groups, which leads to the reduced number of factors.

allowed us to take a clearer look at the influence of communication on group performance. In the German setting, as noted above, groups had a history of working together and were able to self-select which mode of communication to use. Figure 3 shows the scree plot of the two datasets, comparing modes of communication.

The CI factor emerges clearly across communication media, group contexts (i.e., short-term ad hoc vs. long-term) and cultures, explaining about $40 \%$ or more of the variance in all cases. This supports the generality of the metric for studying groups across various modes of communication, group contexts, and cultural settings.

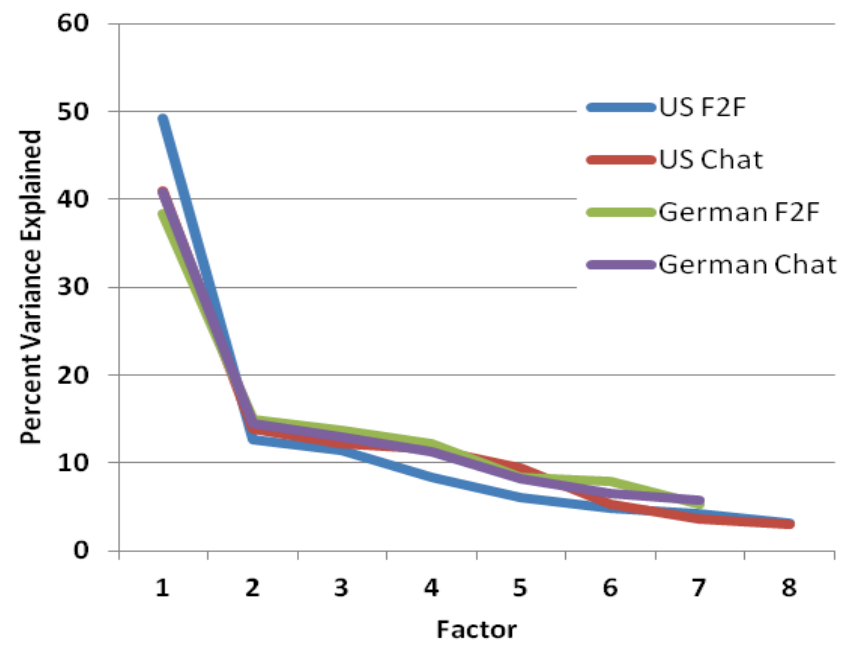

Figure 3: Scree plots of the U.S. and German samples comparing culture and communication media. 


\section{Collective Intelligence is correlated with performance on complex tasks}

In two of our samples, groups worked on other projects or complex tasks as a criterion task, in addition to completing the collective intelligence battery. Their performance on these tasks was moderately correlated with their collective intelligence scores.

In the U.S. lab-based study, both the online and face-to-face teams participated together in the Desert Survival Situation at a later time in the same lab session. Groups completed the task using the same mode of communication that was used to complete the collective intelligence battery.

Analyses of group performance scores on the Desert Survival Situation demonstrated that groups' CI scores were a strong positive predictor of performance on the task ( $b=.24, p=.058)$, controlling for communication media.

In the German student teams, the project descriptions and prototypes were rated by 47 other students along the following dimensions: Novelty, Added Value, Innovativeness, Creativity, Interestingness, Business Relevance and New Thinking. There was a significant positive correlation between the average scores received by student raters and the team's CI scores: $r=.25(p=.008)$. While smaller than the relationship between $\mathrm{CI}$ and performance observed in lab-based studies, it is comparable in size to other relationships between standardized tests and performance in longer-term, multi-faceted situations, such as between SAT scores and individual college grade point average (GPA): $r=.23(p<.01)$ [27]. It is also substantially higher than the average correlation of individual intelligence and group performance found in meta-analyses of field studies: $r=.14,(p>.05)$ [7]. Though we did not have the data to evaluate it here, prior studies have documented a weak relationship between aggregate individual intelligence and collective intelligence, suggesting the former is not a proxy for the latter [43]. Taken together, the results support the criterion validity of our battery as a measure of collective intelligence, which accounts for reasonable variance in team performance both within and outside laboratory.

It is also noteworthy that CI, as measured using the battery of various tasks, is also a much stronger predictor of student team performance than any of the single tasks in the CI battery alone (average $r=.12$ ) except for the most highly correlated task $(r=.27)$. Had we attempted to gauge team performance capability with the use of a single task, as is common in most lab studies, we would have likely done poorly, since all but one of the tasks correlated less with the project score than did the composite CI measure, and it is not possible to know in advance which task will correlate most highly. This demonstrates the advantage of the composite CI score over traditional ways of measuring group performance by using a single task only. Our measure of collective intelligence is more robust against noise than are single tasks, in part because it more reliably measures the underlying factor that drives group performance.

\section{SUMMARY \& CONCLUSION}

We have presented a meta-analysis of three studies investigating the emergence of a general collective intelligence factor in groups working together on a wide array of tasks in our online testing environment. The studies were conducted in three different cultural settings (US, Germany and Japan), in three different languages, using slightly different subsets of tasks and different modes of communication. However, the factor structure that emerges is highly congruent across all three studies. These results suggest some underlying similarities in the basic interaction patterns supporting human group performance, despite differences in communication modes, group contexts, and cultural settings.

While we have shown that the concept of CI is general, questions remain as to whether CI emerges in similar ways across various modes of communication, group contexts, and cultural settings. For example, Woolley et al. [43], in their lab studies with US ad hoc groups interacting face to face, found that CI was positively correlated with group members' average social sensitivity and equal distribution of conversational turn-taking. Engel et al. [8] validated the role of these factors in CI for groups working online. An important question that future research should pursue is whether these factors are as important for groups with ongoing working relationships and for groups in other cultures. For example, groups with ongoing working relationships may have already established a system of who knows what and who does what, in which case distribution of turn-taking is likely to be a manifestation of variance in roles and expertise domains. Thus, in some cases, unequal distribution may demonstrate greater specialization and efficient coordination in long-term groups, which can affect collective intelligence.

In cultures where power distance is considered more natural such as East Asian countries, distribution of participation may not vary as much as in their western counterparts, thereby having limited role in affecting CI. National cultures characterized as high-context cultures may also have higher social sensitivity than low-context cultures on average; thus, the role of social sensitivity in CI may be limited by a ceiling effect. While the current study did not allow us to explore these possibilities, future research can tease out the effects of group contexts and cultural settings on the process of CI emergence.

In addition, we did not have access to individual intelligence scores or grades for the individual participants in these studies, and were not able to determine the correlation between them or the relative contributions of individual intelligence versus collective intelligence to group performance. Earlier work on the topic demonstrated a relatively weak correlation between the two [43]. However, the utility of the concept of collective intelligence 
does not rely upon its distinctiveness from individual intelligence, so much as its ability to capture underlying patterns of behavior in the group driving performance across different domains. These patterns can be influenced by a range of individual differences, including general intelligence and social ability, as well as a host of grouplevel norms, routines, and even collaboration technologies, the latter being of greatest interest to us here.

In closing, we believe that our results have important implications for designers of collaboration technologies. In particular, they support the idea that researchers can use collective intelligence tests to measure the effects of various kinds of general-purpose group collaboration tools, such as email systems, web conferencing software, and social media, on group performance. The CI battery provides a carefully chosen set of tasks to use for testing the effects of such tools on group performance. For instance, a researcher with two versions of a web conferencing system could have groups take our collective intelligence test after randomly assigning the groups to use either version $\mathrm{A}$ or version $\mathrm{B}$ while doing the test. If the groups using version A perform better, then the researcher has a statistical basis for predicting that groups using version A would perform better on a wide range of other tasks, too. This, therefore, provides an economy of effort in testing tools to facilitate online collaboration in a wide variety of settings.

\section{ACKNOWLEDGMENTS}

This work was made possible by financial support from the National Science Foundation (grant numbers IIS-0963285 ACI-1322254, and IIS-0963451), the U. S. Army Research Office (grant numbers 56692-MA and 64079-NS) and Cisco Systems, Inc. through their sponsorship of the MIT Center for Collective Intelligence. We wish to thank Geena Chen, Nada Hashmi and Lisa Jing from MIT, and Sabine Schlick and Lucas Calmbach from University ErlangenNuremberg for their help with data collection.

\section{REFERENCES}

1. Barlow, J.B. and Dennis, A.R. Not as smart as we think: A study of collective intelligence in virtual groups. Proceedings of Collective Intelligence 2014, (2014).

2. Borgatti, S.P. and Cross, R. A relational view of information seeking and learning in social networks. Management Science 49, 4 (2003), 432-445.

3. Carmel, E. Global Software Development Teams: Collaborating across Borders and Time Zones. Prentice Hall, Upper Saddle River, NJ, 1999.

4. Chabris, C.F. Cognitive and neurobiological mechanisms of the law of general intelligence. In M.J. Roberts, ed., Integrating the Mind: Domain General versus Domain specific Processes in Higher Cognition. Psychology Press, Hove, UK, 2007, 449-491.

5. Daft, R.L. and Lengel, R.H. Organizational information requirements, media richness and structural design. Management Science 32, 5 (1986), 554-571.

6. Deary, I.J. Looking Down on Human Intelligence: From Psychometrics to the Brain. Oxford University Press, New York, 2000.

7. Devine, D.J. and Philips, J.L. Do smarter teams do better: A meta-analysis of cognitive ability and team performance. Small Group Research 32, 5 (2001), 507-532.

8. Engel, D., Woolley, A.W., Jing, L.X., Chabris, C.F., and Malone, T.W. Reading the mind in the eyes or reading between the lines? Theory of Mind predicts collective intelligence equally well online and face-toface. PLoS ONE 9, 12 (2014).

9. Gersick, C.J. and Hackman, J.R. Habitual routines in task-performing groups. Organizational Behavior and Human Decision Processes 47, (1990), 65-97.

10. Hall, E.T. Beyond Culture. Anchor Press--Doubleday, New York, 1976.

11. Hertel, G., Konradt, U., and Orlikowsi, B. Managing distance by interdependence: Goal setting, task interdependence and team-based rewards in virtual teams. European Journal of Work and Organizational Psychology 13, (2004), 1-28.

12. Hinds, P. and Reinecke, K. Advancing Methodologies for Cross- Cultural Studies of Collaborative Systems. Proceedings of the Companion Publication of the 17th ACM Conference on Computer Supported Cooperative Work, ACM Press (2014), 323-326.

13. Hofstede, G.H. Culture's Consequences, International Differences in Work-related Values. Sage, Beverly Hills, CA, 1980.

14. Kayan, S., Fussell, S.R., and Setlock, L.D. Cultural differences in the use of instant messaging in Asia and North America. Proceedings of the 2006 20th Anniversary Conference on Computer Supported Cooperative Work, ACM Press (2006), 525-528.

15. Kitayama, S. Culture and basic psychological processes: Toward a system view of culture: Comment on Oyserman et al. (2002). Psychological Bulletin 128, (2002), 89-96.

16. Lafferty, J.C., Pond, A.W., and International, H.S. Desert Survival Situation. Human Synergistics International, 1987.

17. Larson, J.R. In Search of Synergy in Small Group Performance. Psychology Press, New York, NY, 2009.

18. Lepine, J.A., Hollenbeck, J.R., Ilgen, D.R., and Hedlund, J. Effects of individual differences on the performance of hierarchical decision-making teams: Much more than g. Journal of Applied Psychology 82, 5 (1997), 803-811.

19. Lewis, K. Knowledge and performance in knowledgeworker teams: A longitudinal study of transactive memory systems. Management Science 50, (2004), 1519-1533.

20. Lorenzo-Seva, U. and Ten Berge, J.M. Tucker's congruence coefficient as a meaningful index of factor 
similarity. Methodology: European Journal of Research Methods for the Behavioral and Social Sciences 2, 2 (2006), 57-64.

21. Markus, M.L. Electronic mail as the medium of managerial choice. Organization Science 5, 4 (1994), 502-527.

22. Martins, L.L., Gilson, L.L., and Maynard, M.T. Virtual teams: What do we know and where do we go from here? Journal of Management 30, 6 (2004), 805-836.

23. McDonough, E. An investigation of the use of global, virtual, and colocated new product development teams. Journal of Product Innovation Management 18, (2001), 110-120.

24. McGrath, J.E. Groups: Interaction and Performance. Prentice-Hall, Englewood Cliffs, NJ, 1984.

25. Myers, M., D. and Tan, F., B. Beyond models of national culture in information systems research. In Advanced Topics in Global Information Management. Idea Group Inc (IGI), 2002, 14-29.

26. Neuman, G.A., Wagner, S.H., and Christiansen, N.D. The relationship between work-team personality composition and the job performance of teams. Group \& Organization Management 24, (1999), 28-45.

27. Noftle, E.E. and Robins, R.W. Personality predictors of academic outcomes: Big five correlates of GPA and SAT scores. Journal of Personality and Social Psychology 93, 1 (2007), 116-130.

28. Nye, J.L. and Brower, A.M. What's Social about Social Cognition? Research on Socially Shared Cognition in Small Groups. Sage, Thousand Oaks, CA, 1996.

29. Oyserman, D., Coon, H.M., and Kemmelmeier, M. Rethinking individualism and collectivism: Evaluation of theoretical assumptions and meta-analyses. Psychological Bulletin 128, 1 (2002), 3-72.

30. Probst, T.M., Carnevale, P.J., and Triandis, H.C. Cultural values in intergroup and single-group social dilemmas. Organizational Behavior and Human Decision Processes 77, 3 (1999), 171-191.

31. Ren, Y. and Argote, L. Transactive memory systems 1985-2010: An integrative framework of key dimensions, antecedents, and consequences. The Academy of Management Annals 5, 1 (2011), 189-229.
32. Rousseau, D.M. Issues of level in organizational research: Multi-level and cross-level perspectives. In L.L. Cummings and B. Staw, eds., Research in Organizational Behavior. JAI, Greenwich, CT, 1985, $1-37$.

33. Russell, S.J. Artificial Intelligence: A Modern Approach. Prentice Hall, Upper Saddle River, 2010.

34. Scissors, L., Shami, N.S., Ishihara, T., Rohall, S., and Saito, S. Real-time collaborative editing behavior in USA and Japanese distributed teams. Proceedings of the SIGCHI Conference on Human Factors in Computing Systems, ACM Press (2011), 1119-1128.

35. Setlock, L.D., Fussell, S.R., and Neuwirth, C. Taking it out of context: Collaborating within and across cultures in face-to-face settings and via instant messaging. Proceedings of the 2004 ACM Conference on Computer Supported Cooperative Work, ACM Press (2004), 604-613.

36. Short, J., Williams, E., and Christie, B. The Social Psychology of Telecommunications. Wiley, New York, 1976.

37. Smith, E.E. Cognitive Psychology: Mind and Brain. Pearson/Prentice Hall, Upper Saddle River, N.J, 2007.

38. Spearman, C. General intelligence, objectively determined and measured. American Journal of Psychology 15, 2 (1904), 201-293.

39. Tschan, F. Ideal cycles of communication (or cognitions) in triads, dyads, and individuals. Small Group Research 33, 6 (2002), 615-643.

40. Tucker, L.R. A Method of Synthesis of Factor Analysis Studies. Department of the Army, Washington, D.C., 1951.

41. Wagner, J.A. and Moch, M.K. Individualismcollectivism: Concept and measure. Group \& Organization Management 11, 3 (1986), 280-304.

42. Walther, J.B. Interpersonal effects in computermediated interaction: A relational perspective. Communication Research 19, 1 (1992), 52-90.

43. Woolley, A.W., Chabris, C.F., Pentland, A., Hashmi, N., and Malone, T.W. Evidence for a collective intelligence factor in the performance of human groups. Science 330, (2010), 686-688. 